\title{
Environmental-friendly one-pot cascade synthesis of 3-cyanopiperidin-2,6-diones
}

\author{
Daniel Paprocki ${ }^{1} \cdot$ Stanisław Berłożecki ${ }^{1} \cdot$ Ryszard Ostaszewski $^{1}$ (]
}

Received: 12 March 2018 / Accepted: 23 July 2019 / Published online: 5 October 2019

(c) The Author(s) 2019

\begin{abstract}
3-Cyanopiperidin-2,6-diones are key intermediates in the synthesis of several drugs and natural products. We developed onepot synthesis of 3-cyanopiperidin-2,6-diones by Knoevenagel condensation of cyanoacetic acid and aldehydes, followed by addition of cyanoacetic acid, Achmatowicz reaction and decarboxylation. We tested a wide range of aliphatic and aromatic aldehydes. Products were obtained under simple, environmentally friendly conditions and in yields up to 99\%. Substrates were stirred in toluene at reflux, and filtered through short pad of silica gel, and the product was purified by recrystallization.
\end{abstract}

Keywords 3-Cyanopiperidin-2,6-diones · One-pot · Cascade process · Green synthesis $\cdot$ Heterocycles

\section{Introduction}

Piperidine derivatives are convenient building blocks and scaffolds in medicinal chemistry (Eftekhari-Sis and Zirak 2015; Domling 2006). Selected examples of important compounds based on 3-cyanopiperidin-2,6-diones are presented in Fig. 1. This includes: the key intermediate in the synthesis of an antidepressant drug-paroxetine (Buus Lassen et al. 1980; Greenhalgh and Simpkins 2002), the antibacterial compounds (i.e. C-73X1) (Aszalos et al. 1967) or the thalidomide derivatives which recently proved their anticancer activities (Yamada et al. 2006).

3-Cyanopiperidin-2,6-diones were obtained before by the addition of deprotonated ethyl cyanoacetate to the $\alpha, \beta$ unsaturated amides derived from the cinnamic acid analogues (Portnoy 1966). The iridium-catalysed formation of similar glutaric imides was also reported (Takaya et al. 2003). However, this method suffers from the use of environmentally harmful iridium complex as catalyst and harsh reaction conditions. Herein, we decided to check whether it is possible to obtain 3-cyanopiperidin-2,6-dione derivatives in one-pot procedure under environmentally friendly conditions. We have developed a novel, one-pot cascade process, based on the Knoevenagel reaction with cyanoacetic

Ryszard Ostaszewski

ryszard.ostaszewski@icho.edu.pl

1 Institute of Organic Chemistry Polish Academy of Sciences, Kasprzaka 44/52, 01-224 Warsaw, Poland acid and aldehydes. In the second step, the Michael addition of second cyanoacetic acid molecule occurs, followed by the Achmatowicz reaction and subsequent decarboxylation (Achmatowicz et al. 1997). As a result, a wide range of 3-cyanopiperidin-2,6-diones (2, Scheme 1 ) was obtained. The cascade, one-pot reactions are especially beneficial in the viewpoint of green chemistry, while it significantly reduces amount of waste produced during purification of intermediates. Such strategy simplifies the synthetic procedure, reduces time and energy necessary for the reaction comparing to multi-step procedures. Such one-pot processes are very convenient methodology for the synthesis of biologically relevant heterocycles, while it enables the synthesis of the complex scaffolds from simple molecules in a single process (Wagare et al. 2015; Baharfar et al., 2018; Das Sharma et al. 2008; Kidwai and Mothsra 2006). According to our knowledge, such products have never been obtained in single step, so we have established the first example of one-pot procedure for the synthesis of 3-cyanopiperidin2,6-dione derivatives.

\section{Experimental section}

Analytical HPLC, pump: Merck Hitachi L-6000A, column Kromasil C18 $5 \mu \mathrm{m}(250 \times 4.6 \mathrm{~mm})$, detector: Merck Hitachi UV detector L-7400, $\lambda=264 \mathrm{~nm}$, eluent: isocratic mixture of water/methanol $6: 4 \mathrm{v} / \mathrm{v}$, flow: $0.8 \mathrm{ml} / \mathrm{min}$. NMR spectra were measured with a Bruker DRX 500 "Avance" 
Fig. 1 Biologically relevant compounds based on 3-cyanopiperidin-2,6-dione scaffolds<smiles>O=C1CC(c2ccc(F)cc2)C(CO)C(=O)N1</smiles>

Paroxetine intermediate<smiles>[R]C1CC(=O)NC(=O)C1N1C(=O)c2ccccc2C1=O</smiles>

Thalidomide $\left(\mathrm{R}^{1}=\mathrm{H}\right)$<smiles>[R]OC1CC(=O)NC(=O)C1C(=O)O</smiles>

$\mathrm{R}_{2}=4-\mathrm{PO}(\mathrm{OH})_{2} \mathrm{C}_{6} \mathrm{H}_{4}$
Scheme 1. Synthetic strategies leading to formation of 3-cyanopiperidin-2,6-diones. Classical multi-step synthesis $(\cdots)$ versus proposed in this work one-pot cascade process (- - -)

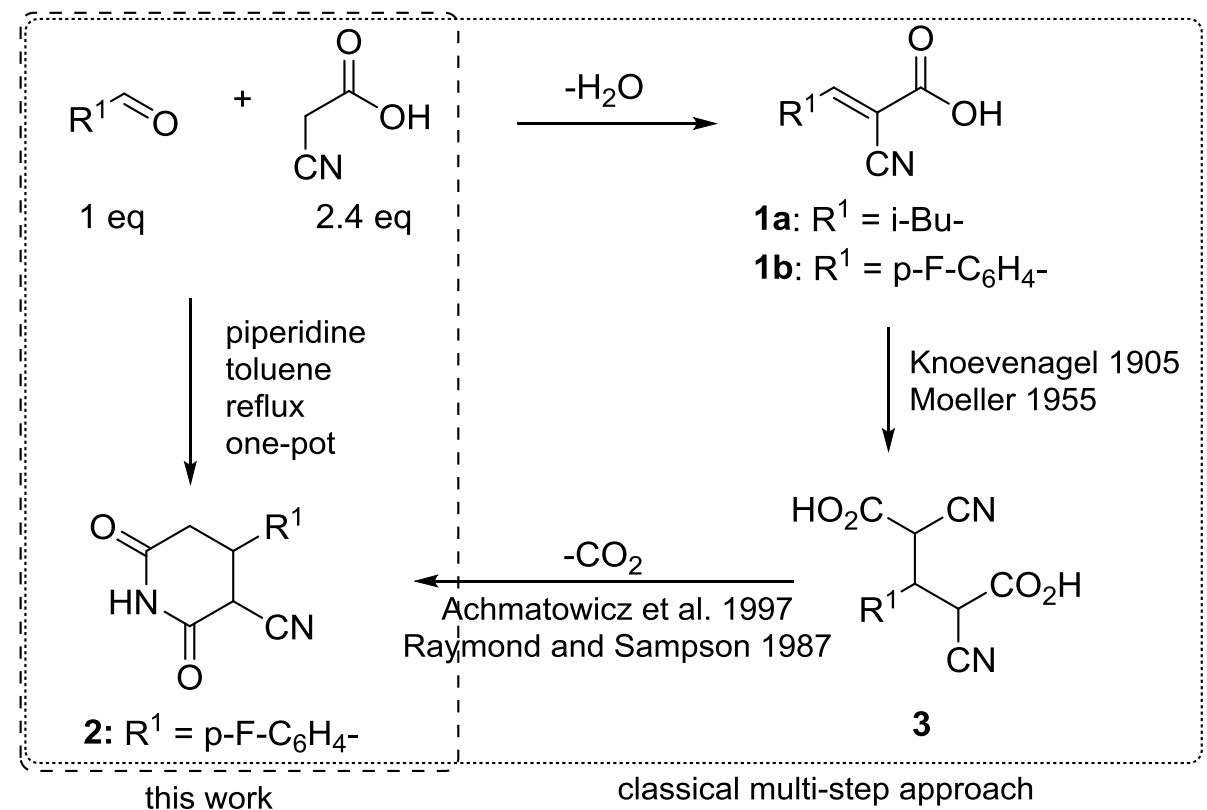

spectrometer with DMS used as an internal standard. CHN analyses were performed on Vario EL Elemental. MS spectra were recorded on an API-365 (SCIEX) apparatus and MS-HR spectra on a Synapt G-2-S (Waters) apparatus. Melting point of products were determined by Boetius apparatus.

\section{General experimental procedure.}

Cyanoacetic acid (1.2 g, $14 \mathrm{mmol})$, appropriate aldehyde $(6 \mathrm{mmol})$ and piperidine $(50 \mu \mathrm{L}, 0.5 \mathrm{mmol})$ were stirred in $2.5 \mathrm{ml}$ of toluene at reflux for $20 \mathrm{~h}$. Toluene was evaporated under reduced pressure, and the residue was dissolved in ethyl acetate and filtered through short pad of silica gel (c.a. $10 \mathrm{~g}$ on Buchner funnel), using hexane-ethyl acetate as eluent. The product was then recrystallized from hexane-ethyl acetate.

4-(4-Fluorophenyl)-2,6-dioxopiperidine-3-carbonitrile (2) White crystals, $\mathrm{mp}=201-209{ }^{\circ} \mathrm{C} ;{ }^{1} \mathrm{H}$ NMR (Major diastereoisomer, $\left.500 \mathrm{MHz}, \mathrm{DMSO}-d_{6}\right): \delta_{\mathrm{H}} 2.61-2.71$ (m, 1H), 2.85-2.95 (m, 1H), 3.82-3.92 (m, 1H), 4.85 (d, $J=12.6 \mathrm{~Hz}, 1 \mathrm{H}), 7.25(\mathrm{t}, J=8.8 \mathrm{~Hz}, 2 \mathrm{H}), 7.41-7.49(\mathrm{~m}$, $2 \mathrm{H}), 11.47(\mathrm{~s}, 1 \mathrm{H}) ;{ }^{13} \mathrm{C}$ NMR $\left(125 \mathrm{MHz}, \mathrm{DMSO}-d_{6}\right): \delta_{\mathrm{C}}$ 37.8, 39.0, 41.7, 115.6, 115.8, 116.0, 129.5, 135.4, 160.7, 162.6, 166.3, 171.0; Anal calcd.: $\mathrm{C}_{12} \mathrm{H}_{9} \mathrm{~N}_{2} \mathrm{O}_{2} \mathrm{~F}: \mathrm{C}, 62.06$; $\mathrm{H}, 3.91 ; \mathrm{N}, 12.07 \%$; found: $\mathrm{C}, 62.11 ; \mathrm{H}, 4.14 ; \mathrm{N}, 12.07 \%$.

4-Methyl-2,6-dioxopiperidine-3-carbonitrile (4) White crystals, $\mathrm{mp}=132-141{ }^{\circ} \mathrm{C} ;{ }^{1} \mathrm{H}$ NMR Diastereisomer1: $\left(500 \mathrm{MHz}, \mathrm{DMSO}-d_{6}\right): \delta_{\mathrm{H}} 1.07(\mathrm{~d}, J=6.9 \mathrm{~Hz}, 3 \mathrm{H}), 2.43$ (dd, $J=17.2,4.3 \mathrm{~Hz}, 1 \mathrm{H}), 2.62-2.68(\mathrm{~m}, 1 \mathrm{H}), 2.76(\mathrm{dd}$, $J=17.3,5.0 \mathrm{~Hz}, 1 \mathrm{H}), 4.59$ (d, $J=4.7 \mathrm{~Hz}, 1 \mathrm{H}), 11.32$ (br. s, $1 \mathrm{H})$; Diastereoisomer2: ${ }^{1} \mathrm{H}$ NMR $\left(500 \mathrm{MHz}, \mathrm{DMSO}-d_{6}\right): \delta_{\mathrm{C}}$ $1.13(\mathrm{~d}, J=6.3 \mathrm{~Hz}, 3 \mathrm{H}), 2.28-2.39(\mathrm{~m}, 1 \mathrm{H}), 2.53-2.61(\mathrm{~m}$, 2H), 4.23 (d, $J=12.0 \mathrm{~Hz}, 1 \mathrm{H}), 11.27$ (br. s, $1 \mathrm{H}) ;{ }^{13} \mathrm{C} \mathrm{NMR}$ $\left(125 \mathrm{MHz}, \mathrm{DMSO}-d_{6}\right): \delta_{\mathrm{C}} 15.8,18.6,27.2,28.4,37.0,38.1$, 40.6, 42.1, 116.4, 116.2, 166.1, 166.8 171.4, 171.5; Anal. calcd for: $\mathrm{C}_{7} \mathrm{H}_{8} \mathrm{~N}_{2} \mathrm{O}_{2}: \mathrm{C}, 55.26 ; \mathrm{H}, 5.30 ; 18.41 \%$; found: $\mathrm{C}$, $55.16 ; \mathrm{H}, 5.47 ; \mathrm{N}, 18.27 \%$. 
4-Ethyl-2,6-dioxopiperidine-3-carbonitrile (5) White crystals, $\mathrm{mp}=122-126{ }^{\circ} \mathrm{C} ;{ }^{1} \mathrm{H}$ NMR $(500 \mathrm{MHz}, \mathrm{DMSO}-$ $\left.d_{6}\right): \delta_{\mathrm{H}} 0.87-0.96(\mathrm{~m}, 11 \mathrm{H}), 1.23-1.32(\mathrm{~m}, 1 \mathrm{H}), 1.33-1.43$ $(\mathrm{m}, 3 \mathrm{H}), 1.58(\mathrm{dt}, J=12.6,6.6 \mathrm{~Hz}, 1 \mathrm{H}), 1.66-1.76(\mathrm{~m}, 3 \mathrm{H})$, $2.28-2.36(\mathrm{~m}, 3 \mathrm{H}), 2.38-2.48(\mathrm{~m}, 4 \mathrm{H}), 2.59$ (dd, $J=16.7$, $3.8 \mathrm{~Hz}, 3 \mathrm{H}), 2.71(\mathrm{dd}, J=17.3,4.4 \mathrm{~Hz}, 1 \mathrm{H}), 4.28$ (d, $J=12.0 \mathrm{~Hz}, 3 \mathrm{H}), 4.57$ (d, $J=4.4 \mathrm{~Hz}, 1 \mathrm{H}), 11.25$ (br. s, 2H), 11.30 (br. s, $1 \mathrm{H}) ;{ }^{13} \mathrm{C}$ NMR (125 MHz, DMSO- $\left.d_{6}\right): \delta_{\mathrm{C}}$ 9.4, 10.9, 23.2, 25.3, 33.2, 25.3, 33.2, 33.7, 34.1, 35.1, 39.8, 40.7, 116.3, 115.9, 166.0, 166.8, 171.3, 171.6; Anal. Calcd: for: $\mathrm{C}_{8} \mathrm{H}_{10} \mathrm{~N}_{2} \mathrm{O}_{2}$ : C, 57.82; $\mathrm{H}, 6.07 ; \mathrm{N}, 16.86 \%$; found: $\mathrm{C}$, $57.76 ; \mathrm{H}, 6.03 ; \mathrm{N}, 17.03 \%$.

4-Butyl-2,6-dioxopiperidine-3-carbonitrile (6) White crystals, $\mathrm{mp}=113-115^{\circ} \mathrm{C} ;{ }^{1} \mathrm{H}$ NMR $\left(500 \mathrm{MHz}, \mathrm{DMSO}-d_{6}\right)$, 3:1 mixture of diastereoisomers: $\delta_{\mathrm{H}} 0.84-0.96(\mathrm{~m}, 3 \mathrm{H}+1 \mathrm{H})$, $1.22-1.33(\mathrm{~m}, 0.33 \mathrm{H}), 1.33-1.43(\mathrm{~m}, 1 \mathrm{H}), 1.53-1.63(\mathrm{~m}$, $0.33 \mathrm{H}), 1.66-1.76(\mathrm{~m}, 1 \mathrm{H}), 2.28-2.36(\mathrm{~m}, 1 \mathrm{H}), 2.38-2.48$ $(\mathrm{m}, 1 \mathrm{H}+0,66 \mathrm{H}), 2.59(\mathrm{dd}, J=16.7,3.8 \mathrm{~Hz}, 1 \mathrm{H}), 2.71(\mathrm{dd}$, $J=17.3,4.4 \mathrm{~Hz}, 0,33 \mathrm{H}), 4.28(\mathrm{~d}, J=12.0 \mathrm{~Hz}, 1 \mathrm{H}), 4.57$ $(\mathrm{d}, J=4.4 \mathrm{~Hz}, 0.33 \mathrm{H}), 11.25$ (br. s., $1 \mathrm{H}+0.33 \mathrm{H}) ;{ }^{13} \mathrm{C} \mathrm{NMR}$ $\left(125 \mathrm{MHz}, \mathrm{DMSO}-d_{6}\right): \delta_{\mathrm{C}} 13.7,21.9,22.0,26.8,28.0,29.7$, 31.6, 32.2, 32.4, 34.5, 35.7, 41.1, 115.9, 116.4, 166.1, 166.8, 171.3, 171.6 Anal calcd.: $\mathrm{C}_{10} \mathrm{H}_{14} \mathrm{~N}_{2} \mathrm{O}_{2}$ : C, 61.82; H, 7.28; N, 14.42\%; found: C, 61.52; H, 7.40; N, 14.63\%; LR-MS (ESI, $\left[\mathrm{M}+\mathrm{Na}^{+}\right]$) calcd for: $\mathrm{C}_{10} \mathrm{H}_{14} \mathrm{~N}_{2} \mathrm{O}_{2} \mathrm{Na}: 217$, found, 217.

\section{4-(2-Methylpropyl)-2,6-dioxopiperidine-3-carboni-} trile (7) ${ }^{1} \mathrm{H}$ NMR (Mixture of diastereoisomers, $500 \mathrm{MHz}$, DMSO- $\left.d_{6}\right): \delta_{\mathrm{H}} 0.78-0.87(\mathrm{~m}, 3 \mathrm{H}+1.5 \mathrm{H}), 0.88-0.94(\mathrm{~m}$, $3 \mathrm{H}+1.5 \mathrm{H}), 1.17-1.25(\mathrm{~m}, 0.5 \mathrm{H}), 1.25-1.33(\mathrm{~m}, 1 \mathrm{H}+0.5 \mathrm{H})$, $1.35-1.43(\mathrm{~m}, 1 \mathrm{H}), 1.61-1.75 \delta_{\mathrm{C}}(\mathrm{m}, 1 \mathrm{H}+0.5 \mathrm{H}), 2.23-2.31$ $(\mathrm{m}, 1 \mathrm{H}), 2.44(\mathrm{dd}, J=17.3,5.9 \mathrm{~Hz}, 0.5 \mathrm{H}), 2.52-2.63(\mathrm{~m}$, 2H), 2.69 (dd, $J=17.3,4.5 \mathrm{~Hz}, 0.5 \mathrm{H}), 4.24(\mathrm{~d}, J=11.9 \mathrm{~Hz}$, 1H), 4.54 (d, $J=4.5 \mathrm{~Hz}, 0.5 \mathrm{H}$ ), 11.27 (br. s., 1H), 11.32 (br. s., $0.5 \mathrm{H}) ;{ }^{13} \mathrm{C} \mathrm{NMR}$ (Mixture of diastereoisomers, $125 \mathrm{MHz}$, DMSO- $\left.d_{6}\right): \delta_{\mathrm{C}} 21.4,22.3,22.9,23.8,25.1,31.0,32.4,35.2$, 36.3, 40.5, 42.3, 42.9, 113.7, 114.8, 164.5, 165.0, 170.3, 170.5; Anal calcd.: $\mathrm{C}_{10} \mathrm{H}_{14} \mathrm{~N}_{2} \mathrm{O}_{2}: \mathrm{C}, 61.84 ; \mathrm{H}, 7.27 ; \mathrm{N}$, $14.42 \%$; found: C, $61.60 ; \mathrm{H}, 7.43 ; \mathrm{N}, 14.33 \%$.

4-Octyl-2,6-dioxopiperidine-3-carbonitrile (8) White crystals, $\mathrm{mp}=115-126{ }^{\circ} \mathrm{C} ;{ }^{1} \mathrm{H}$ NMR (Mixture of diastereoisomers, $\left.500 \mathrm{MHz}, \mathrm{DMSO}-d_{6}\right): \delta_{\mathrm{H}} 0.79-0.96(\mathrm{~m}$, $3 \mathrm{H}+3 \times 0.33 \mathrm{H}), 1.15-1.42(\mathrm{~m}, 18 \mathrm{H}), 1.51(\mathrm{~m}, 0.33 \mathrm{H})$, 1.57-1.70 (m, 1H), 2.24-2.39 (m, 1H), 2.41-2.48 (m, $1 \mathrm{H}+0.33 \mathrm{H}), 2.58(\mathrm{dd}, J=16.7,3.8 \mathrm{~Hz}, 1 \mathrm{H}), 2.65-2.77$ $(\mathrm{m}, 0.33 \mathrm{H}), 4.27(\mathrm{~d}, J=12.0 \mathrm{~Hz}, 1 \mathrm{H}), 4.56(\mathrm{~d}, J=3.5 \mathrm{~Hz}$, $0.33 \mathrm{H}), 11.16-11.34(\mathrm{~m}, 1 \mathrm{H}+0.33 \mathrm{H}) ;{ }^{13} \mathrm{C} \mathrm{NMR}(125 \mathrm{MHz}$, DMSO- $\left.d_{6}\right): \delta_{\mathrm{C}} 13.3,22.0,24.6,25.8,28.5,28.7,28.8,30.0$, 31.2, 31.6, 32.4, 32.5, 41.1, 115.8, 116.3, 166.0, 166.73, 171.3, 171.5; Anal calcd.: $\mathrm{C}_{14} \mathrm{H}_{22} \mathrm{~N}_{2} \mathrm{O}_{2}$ : C, 67.17; H, 8.86; N, $11.19 \%$; found: C, 67.03; H, 8.93; N, 11.19\%.
4-(Non-8-en-1-yl)-2,6-dioxopiperidine-3-carbonitrile (9) white crystals, $\mathrm{mp}=116-119{ }^{\circ} \mathrm{C} ;{ }^{1} \mathrm{H}$ NMR (mixture of diastereoisomers 1:2.2, $\left.500 \mathrm{MHz}, \mathrm{DMSO}-d_{6}\right): \delta_{\mathrm{H}} 1.20-1.30$ (m, 14H), 1.32-1.41 (m, 6H), 1.47-1.54 (m, 0.4H), 1.59$1.66(\mathrm{~m}, 1 \mathrm{H}), 1.97-2.04(\mathrm{~m}, 3 \mathrm{H}), 2.28-2.32(\mathrm{~m}, 1 \mathrm{H}), 2.40$ $2.49(\mathrm{~m}, 1.5 \mathrm{H}), 2.58(\mathrm{dd}, J=16.9,3.9 \mathrm{~Hz}, 1 \mathrm{H}), 2.67-2.74$ $(\mathrm{m}, 0.4 \mathrm{H}), 4.26(\mathrm{~d}, J=12.3 \mathrm{~Hz}, 1 \mathrm{H}), 4.56(\mathrm{~d}, J=4.1 \mathrm{~Hz}$, $0.4 \mathrm{H}), 4.90-5.02(\mathrm{~m}, 2.9 \mathrm{H}), 5.74-5.84(\mathrm{~m}, 1.4 \mathrm{H}), 11.25$ $(\mathrm{s}, 1 \mathrm{H}), 11.30(\mathrm{~s}, 0.4 \mathrm{H}) ;{ }^{13} \mathrm{C}$ NMR $\left(125 \mathrm{MHz}, \mathrm{DMSO}-d_{6}\right)$ : $\delta_{\mathrm{C}} 24.5,25.8,28.2,28.4,28.6,28.7,28.7,28.8,30.0,31.6$, $32.4,32.5,33.134 .5,35.6,41.0,114.5,115.8,116.3,138.8$, 166.0, 166.7, 171.2, 171.5; Anal calcd.: $\mathrm{C}_{16} \mathrm{H}_{24} \mathrm{~N}_{2} \mathrm{O}_{2}$ : C, $69.53 ; \mathrm{H}, 8.75 ; \mathrm{N}, 10.14 \%$; found: C, 69.40; H, 8.78; N, $9.92 \%$

2,6-Dioxo-4-phenylpiperidine-3-carbonitrile (10) white crystals, $\mathrm{mp}=222-224{ }^{\circ} \mathrm{C} ;{ }^{1} \mathrm{H}$ NMR (Major diastereoisomer, $\left.500 \mathrm{MHz}, \mathrm{DMSO}-d_{6}\right): \delta_{\mathrm{H}} 2.65(\mathrm{dd}$, $J=17.1,4.3 \mathrm{~Hz}, 1 \mathrm{H}), 2.86-2.94(\mathrm{~m}, 1 \mathrm{H}) 3.83(\mathrm{td}, J=12.8$, $4.3 \mathrm{~Hz}, 1 \mathrm{H}) 4.87(\mathrm{~d}, J=12.7 \mathrm{~Hz}, 1 \mathrm{H}) 7.31-7.37(\mathrm{~m}, 1 \mathrm{H})$ 7.37-7.45 (m, 3H) $11.46(\mathrm{~s}, 1 \mathrm{H}){ }^{13} \mathrm{C}$ NMR (Major diastereoisomer, $125 \mathrm{MHz}$, DMSO- $\left.d_{6}\right): \delta_{\mathrm{C}} 38.9,39.1,42.03,116.5$, 127.8, 128.4, 129.4, 139.6, 166.9, 171.5; HR-MS (EI, [M ${ }^{+}$]) calcd for: $\mathrm{C}_{12} \mathrm{H}_{10} \mathrm{~N}_{2} \mathrm{O}_{2}: 214.0742$, found, 214.0733; Anal calcd.: $\mathrm{C}_{12} \mathrm{H}_{10} \mathrm{~N}_{2} \mathrm{O}_{2}$ : C, 67.28; $\mathrm{H}, 4.71 ; \mathrm{N}, 13.08 \%$; found: $\mathrm{C}$, $66.93 ; \mathrm{H}, 4.64 ; \mathrm{N}, 12.83 \%$.

4-(4-Hydroxyphenyl)-2,6-dioxopiperidine-3-carbonitrile (11) White crystals, $\mathrm{mp}=210-212{ }^{\circ} \mathrm{C},{ }^{1} \mathrm{H}$ NMR (Major diastereoisomer, $\left.500 \mathrm{MHz}, \mathrm{DMSO}-d_{6}\right): \delta_{\mathrm{H}} 2.58(\mathrm{dd}$, $J=17.0,4.1 \mathrm{~Hz}, 1 \mathrm{H}) 2.83(\mathrm{dd}, J=16.9,13 . \mathrm{z}, 1 \mathrm{H}) 3.68$ $(\mathrm{td}, J=12.8,4.1 \mathrm{~Hz}, 1 \mathrm{H}) 4.74(\mathrm{~d}, J=12.6 \mathrm{~Hz}, 1 \mathrm{H}) 6.77(\mathrm{~d}$, $J=8.2 \mathrm{~Hz}, 2 \mathrm{H}) 7.17$ (d, $J=8.5 \mathrm{~Hz}, 2 \mathrm{H}) 9.48(\mathrm{~s}, 1 \mathrm{H}) 11.41$ (br. s., $1 \mathrm{H}) ;{ }^{13} \mathrm{C}$ NMR $\left(125 \mathrm{MHz}, \mathrm{DMSO}-d_{6}\right): \delta_{\mathrm{C}} 38.0,38.8$, 42.1, 115.6, 116.2, 128.3, 129.3, 157.0, 166.6, 171.2; Anal calcd.: $\mathrm{C}_{12} \mathrm{H}_{10} \mathrm{~N}_{2} \mathrm{O}_{3}$ : C, 62.61; $\mathrm{H}, 4.38 ; \mathrm{N}, 12.17 \%$; found: C, 62.56; H, 4.44; N, $11.98 \%$.

4-(4-Methoxyphenyl)-2,6-dioxopiperidine-3-carbonitrile (12) White crystals, $\mathrm{mp}=202-204{ }^{\circ} \mathrm{C} ;{ }^{1} \mathrm{H}$ NMR (Major diastereoisomer, $\left.500 \mathrm{MHz}, \mathrm{DMSO}-d_{6}\right): \delta_{\mathrm{H}} 2.60(\mathrm{dd}$, $J=17.0,4.4 \mathrm{~Hz}, 1 \mathrm{H}) 2.86(\mathrm{dd}, J=17.2,13.1 \mathrm{~Hz}, 1 \mathrm{H})$ $3.33(\mathrm{~s}, 1 \mathrm{H}) 3.76(\mathrm{~s}, 3 \mathrm{H}) 4.80(\mathrm{~d}, J=12.6 \mathrm{~Hz}, 1 \mathrm{H}) 6.96$ $(\mathrm{d}, J=8.8 \mathrm{~Hz}, 2 \mathrm{H}) 7.31(\mathrm{~d}, J=8.5 \mathrm{~Hz}, 2 \mathrm{H}) 11.44(\mathrm{~s}, 1 \mathrm{H})$; ${ }^{13} \mathrm{C}$ NMR (Major diastereoisomer, $125 \mathrm{MHz}, \mathrm{DMSO}-d_{6}$ ): $\delta_{\mathrm{C}} 37.9,38.7,41.9,55.1,114.3,116.1,128.4,131.1,158.8$, 166.5, 171.1; Anal calcd.: $\mathrm{C}_{13} \mathrm{H}_{12} \mathrm{~N}_{2} \mathrm{O}_{3}$ : C, 63.93; $\mathrm{H}, 4.95$; N, $11.47 \%$; found: C, 63.98; H, 5.09; N, $11.27 \%$.

4-(Naphthalen-1-yl)-2,6-dioxopiperidine-3-carbonitrile (13) White crystals $\mathrm{mp}=198-206{ }^{\circ} \mathrm{C}$; ${ }^{1} \mathrm{H}$ NMR $\left(500 \mathrm{MHz}, \mathrm{DMSO}-d_{6}\right): \delta_{\mathrm{H}} 2.72(\mathrm{dd}, J=17.0,4.1 \mathrm{~Hz}, 1 \mathrm{H})$ 2.82-2.95 (m, 1H) 4.84-4.96 (m, 1H) $5.15(\mathrm{~d}, J=12.6 \mathrm{~Hz}$, 


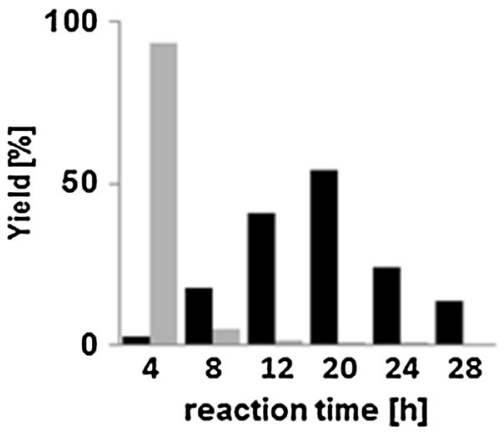

Fig. 2 The yield of the compounds $\mathbf{1 b}$ (grey bar) and $\mathbf{2}$ (black bar) during the reaction of cyanoacetic acid and p-FBA

1H) 7.54-7.64 (m, 3H) $7.68(\mathrm{~d}, J=6.9 \mathrm{~Hz}, 1 \mathrm{H}) 7.90-7.96$ $(\mathrm{m}, 1 \mathrm{H}) 7.96-8.02(\mathrm{~m}, 1 \mathrm{H}) 8.36(\mathrm{~d}, J=8.2 \mathrm{~Hz}, 1 \mathrm{H}) 11.54$ $(\mathrm{s}, 1 \mathrm{H}) ;{ }^{13} \mathrm{C}$ NMR $\left(125 \mathrm{MHz}, \mathrm{DMSO}-d_{6}\right): \delta_{\mathrm{C}} 32.7,38.2$, 41.1, 116.3, 123.0, 123.5, 125.6, 126.1, 126.6, 128.1, 128.8, 131.0, 133.5, 135.6, 166.7, 171.0; Anal calcd.: $\mathrm{C}_{16} \mathrm{H}_{12} \mathrm{~N}_{2} \mathrm{O}_{2}$ : C, 72.72; H, 4.58; N, 10.60\%; found: C, 72.82; H, 4.39; N, $10.62 \%$.

\section{Results and discussion}

For the model studies, $p$-fluorobenzaldehyde (pFBA) and isovaleraldehyde were used as the aldehyde components. The reaction was initially performed under conditions described by Knoevenagel with Dean-Stark apparatus to remove the formation of water. The reaction resulted in formation of compounds $\mathbf{1 a}$ and $\mathbf{1 b}$ (depending on the aldehyde used), what is consistent with literature data (Knoevenagel 1905). Then, we performed the reaction with pFBA without water removal. We found that after $4 \mathrm{~h}$, the compound $\mathbf{1 b}$ was the main product although further transformation of the compound $\mathbf{1 b}$ occurred when the reaction was continued (Fig. 2). After $8 \mathrm{~h}$ only the small amount of $\mathbf{1 b}$ was observed in the reaction mixture, while $17 \%$ of 2 was formed. After $12 \mathrm{~h}$, only trace of $\mathbf{1 b}$ was found and $40 \%$ of $\mathbf{2}$ was present in the reaction. After $20 \mathrm{~h}$ of the reaction, the corresponding 3-cyanopiperidin-2,6-dione $\mathbf{2}$ was isolated as the main product in 54\% yield. Longer reaction time resulted in decomposition of the compound $\mathbf{2}$ and lead to a complex reaction mixture.

Those experiments allowed us to develop a new cascade process, which results in the formation of compound $\mathbf{2}$ in one step. The Michael addition of cyanoacetic acid to the $\alpha, \beta$-unsaturated acid leads to the formation of intermediate 3 which subsequently undergoes the cyclization and decarboxylation reactions. The plausible mechanism of reactions cascade is presented in Table 1. The influence of solvent was also studied, but the experiments in water gave products with yield up to $15 \%$. Additionally, for the reaction with pFBA, the product was obtained as 10:1 diastereoisomeric mixture (determined by ${ }^{1} \mathrm{HNMR}$ ). In Table 1 , we present the scope and substrate limitations of the process. Either aromatic or aliphatic aldehydes can be used, and in all cases the respective 3-cyanopiperidin-2,6-dione derivative has been obtained. Studying the scope of aldehydes, we noticed that in the case of aryl aldehydes, longer reaction time is required (Table 1, entries 7-11). On the other hand, faster reaction rates for aliphatic aldehydes result in significantly lower diastereoisomeric ratios of obtained products 4-9 (entries 1-6). Nevertheless, the reaction is still very convenient and simple method for the preparation of a wide range of this class of compounds.

Reactions conditions: Cyanoacetic acid ( $1.2 \mathrm{~g}, 14 \mathrm{mmol})$, aldehyde $(6 \mathrm{mmol})$ and piperidine $(50 \mu \mathrm{L}, 0.5 \mathrm{mmol})$ were stirred in $2.5 \mathrm{ml}$ of toluene at reflux for $20 \mathrm{~h}$.

\section{Conclusions}

In conclusion, we developed the novel one-pot cascade process for the synthesis of 3-cyanopiperidin-2,6-diones from aldehydes and cyanoacetic acid with piperidine as a catalyst. Given the fact that the presented cascade includes four steps, the obtained yields of the reaction are very good. The process can be performed with various aryl and aliphatic aldehydes. For aromatic aldehydes, the products were obtained with very good yields and with high diastereoselectivity. Moreover, developed process exhibits high atom efficiency, since only by-products are water and carbon dioxide molecules as a result of reaction between threes substrate molecules. Overall, this methodology demonstrates the remarkable potential of acid-base catalysis for the synthesis of complex heterocyclic compounds. This method is attractive, especially from the point of view green chemistry, due to its simplicity, ease of workability, high yields and replacement of multi-step synthesis by one-pot cascade process. 
Table 1 The scope of aldehydes used in reaction, plausible mechanism or reactions cascade above table<smiles>[R]C=C(C)C(=O)OCC(=O)O</smiles>

1<smiles>[R]C(C(C)CCCCCCCCC(=O)O)C(C#N)C(=O)O</smiles>

3<smiles>[R]C1CC(=O)NC(=O)C1C#N</smiles>

\section{2, 4-13}

\begin{tabular}{|c|c|c|c|c|c|}
\hline Entry & R- & Product & Yield $^{\mathrm{a}}, 4 \mathrm{~h}(\%)$ & Yield $^{\mathrm{a}}, 20 \mathrm{~h}(\%)$ & d.r. ${ }^{b}$ \\
\hline 1 & Me- & 4 & 27 & 11 & $1: 3$ \\
\hline 2 & Et- & 5 & 52 & 44 & $1: 3$ \\
\hline 3 & $n$-Butyl- & 6 & 61 & 99 & $1: 3$ \\
\hline 4 & $i$-Butyl- & 7 & 33 & 74 & $1: 2$ \\
\hline 5 & $n-\mathrm{C}_{8} \mathrm{H}_{11^{-}}$ & 8 & 49 & 71 & $1: 2.5$ \\
\hline 6 & $n$-dec-9-enyl- & 9 & 61 & 75 & $\mathrm{n} / \mathrm{d}^{\mathrm{c}}$ \\
\hline 7 & Ph- & 10 & $<1$ & 64 & $1: 10$ \\
\hline 8 & & 11 & $<1$ & 37 & $1: 10$ \\
\hline 9 & & 12 & $<1$ & 24 & $1: 10$ \\
\hline 10 & & 2 & $<1$ & 54 & $1: 10$ \\
\hline 11 & & 13 & $<1$ & 47 & $1: 10$ \\
\hline
\end{tabular}

Reactions conditions: Cyanoacetic acid $(1.2 \mathrm{~g}, 14 \mathrm{mmol})$, aldehyde $(6 \mathrm{mmol})$ and piperidine $(50 \mu \mathrm{L}, 0.5 \mathrm{mmol})$ were stirred in $2.5 \mathrm{ml}$ of toluene at reflux for $20 \mathrm{~h}$

${ }^{\mathrm{a}}$ Isolated yields

${ }^{b}$ Determined by ${ }^{1} \mathrm{HNMR}$

${ }^{\mathrm{c}}$ The determination of d.r. was not possible due to overlapping of the crucial signals

Acknowledgements The authors are grateful to National Science Center, Poland, for financial support, Project OPUS No. 2016/23/B/ ST5/03307

Open Access This article is distributed under the terms of the Creative Commons Attribution 4.0 International License (http://creativecommons. org/licenses/by/4.0/), which permits unrestricted use, distribution, and reproduction in any medium, provided you give appropriate credit to the original author(s) and the source, provide a link to the Creative Commons license, and indicate if changes were made.

\section{References}

Achmatowicz O, Malinowska I, Szechner B, Maurin JK (1997) Resolution of 4-cyano-4-(4-nitrophenyl)hexanoic acid: Synthesis of (R) and (S)-3-(4-aminophenyl)-3-ethylpiperidine-2,6-dione (aminoglutethimide). Tetrahedron 53:7917-7928. https://doi. org/10.1016/S0040-4020(97)00464-X

Aszalos A, Hoberecht H, Cohen AI (1967) Structure of antibiotic C-73X. J Med Chem 10:281-284. https://doi.org/10.1021/jm003 $14 \mathrm{a} 044$ 
Baharfar R, Azimi R, Asdollahpour Z (2018) Efficient microwaveassisted diastereoselective synthesis of indole-based 4,5-dihydrofurans via a one-pot, three-component reaction in water. Environ Chem Lett. https://doi.org/10.1007/s10311-017-0686-3

Buus Lassen J, Lund J, Sondergaard I (1980) Central and peripheral 5-HT uptake in rats treated chronically with femoxetine, paroxetine, and chlorimipramine. Psychopharmacology 68:229-233. https://doi.org/10.1007/BF00428108

Das Sharma S, Hazarika P, Konwar D (2008) An efficient and onepot synthesis of 2,4,5-trisubstituted and 1,2,4,5-tetrasubstituted imidazoles catalyzed by $\mathrm{InCl}_{3} \cdot 3 \mathrm{H}_{2} \mathrm{O}$. Tetrahedron Lett 49:22162220. https://doi.org/10.1016/j.tetlet.2008.02.053

Domling A (2006) Recent developments in isocyanide based multicomponent reactions in applied chemistry. Chem Rev 106:1789. https://doi.org/10.1021/cr0505728

Eftekhari-Sis B, Zirak M (2015) Chemistry of $\alpha$-oxoesters: a powerful tool for the synthesis of heterocycles. Chem Rev 115:151264. https://doi.org/10.1021/cr5004216

Greenhalgh DA, Simpkins NS (2002) A novel chiral base mediated glutarimide desymmetrisation: application to the asymmetric synthesis of (-)-paroxetine. Synlett 12:2074-2076. https://doi. org/10.1055/s-2002-35588

Kidwai M, Mothsra P (2006) A one-pot synthesis of 1,2,4,5-tetraarylimidazoles using molecular iodine as an efficient catalyst. Tetrahedron Lett 47:5029-5031. https://doi.org/10.1016/j.tetle t.2006.05.097

Knoevenagel E (1905). Patent: DE156560

Moeller F (1955) Verfahren zur Herstellung von, '-Dicyan- -alkylglutarsaeuren. Patent: DE924386
Plevey RG, Sampson P (1987) The synthesis of 3-(4-aminotetrafluorophenyl)-3-ethylpiperidine-2,6-dione; a fluorinated derivative of aminoglutethimide. J Chem Soc Perkin Trans 1:2129-2136. https://doi.org/10.1039/P19870002129

Portnoy S (1966) Fluorinated nitrogen heterocycles via cyclization. II. 3 -Trifluoromethylglutarimides from fluorinated $\alpha, \beta$-unsaturated carbonyls and cyanoacetamide. J Het Chem 3:363-364. https:// doi.org/10.1002/jhet.5570030330

Takaya H, Yoshida K, Isozaki K, Terai H, Murahashi SI (2003) Transition-metal-based lewis acid and base ambiphilic catalysts of iridium hydride complexes: multicomponent synthesis of glutarimides. Angew Chem Int Ed 42:3302-3304. https://doi.org/10.1002/ anie. 200351689

Wagare DS, Netankar PD, Shaikh M, Farooqui M, Durrani A (2015) Highly efficient microwave-assisted one-pot synthesis of 4-aryl2-aminothiazoles in aqueous medium. Environ Chem Lett 15:475479. https://doi.org/10.1007/s10311-017-0619-1

Yamada T, Okada T, Sakaguchi K, Ohfune Y, Ueki H, Soloshonok VA (2006) Efficient asymmetric synthesis of novel 4-substituted and configurationally stable analogues of thalidomide. Org Lett 8:5625-5628. https://doi.org/10.1021/o10623668

Publisher's Note Springer Nature remains neutral with regard to jurisdictional claims in published maps and institutional affiliations. 